\title{
Invalidità ed esenzione dai ticket. Ecco come fare
}

\section{G. Sandrini}

Consigliere e Segretaria Generale AIRP

info.airp@renepolicistico.it

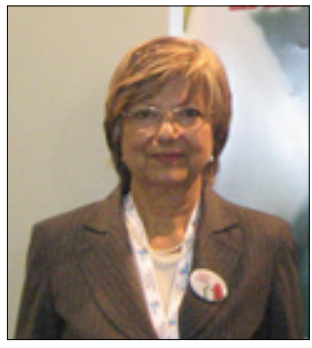

Grazia Sandrini
Un malato di rene policistico ha diritto all'invalidità e all'esenzione del ticket?

La malattia renale policistica non gode delle esenzioni di cui al decreto istitutivo dei cosiddetti L.E.A. (livelli essenziali di assistenza) in quanto, inspiegabilmente, non è considerata una malattia cronica invalidante, se non quando evolve nell'insufficienza renale.

Esiste però un iter per poter ottenere una esenzione parziale per medicinali e una esenzione totale per analisi e indagini specialistiche e strumentali.

\section{Premesso:}

- che il Decreto Ministeriale 5 febbraio 1992 approva la nuova tabella indicativa delle percentuali d'invalidità per le minorazioni e malattie invalidanti (G.U. Serie Generale n. 47 del 26 febbraio 1992);

- che la tabella attribuisce alla malattia del "Rene Policistico Bilaterale", al codice “6480", il 70\% di invalidità fisso;

è possibile richiedere l'invalidità che, in base alla normativa regionale, se superiore al $67 \%$, da diritto appunto all'esenzione sopra indicata.

La procedura non è automatica, richiede qualche passaggio, e precisamente:

- Recarsi dal proprio medico di base, che dovrà redigere un certificato medico telematico apposito per richiesta di invalidità. Sul certificato dovrà essere indicato chiaramente che il paziente soffre di malattia renale policistica e andranno descritte chiaramente tutte le altre patologie eventuali e correlate. - Il medico invia telematicamente una copia del certificato all'INPS e l'originale viene consegnato al paziente. - Il paziente deve recarsi con il certificato presso un patronato (50 \& più, ACLI ecc.) che gratuitamente redige la domanda di invalidità e la invia all'INPS.

- Entro 30 o 60 giorni, il paziente viene convocato dalla Commissione medica che deve valutare l'effettivo stato di salute: in quell'occasione è bene ripetere chiaramente che si è affetti da malattia genetica renale policistica.

- La Commissione deve assegnare il 70\% d'invalidità anche senza riduzione della funzionalità renale, ed è possibile che la percentuale possa essere anche superiore al $70 \%$ in considerazione di eventuali altri problemi di salute.

- Entro breve tempo verrà comunicata la decisione della Commissione, dopodiché si potrà ritirare presso la ASL di competenza il tesserino di esenzione da utilizzare presso il proprio medico di base per tutte le prescrizioni.

Si precisa che contro la decisione della Commissione si potrà fare ricorso entro i termini indicati nella lettera.

Si consiglia inoltre di informarsi preventivamente, presso l'ASL di competenza, se la normativa della propria regione prevede l'esenzione in caso di invalidità superiore al $67 \%$. 